\title{
BANCO E CHUVA DE SEMENTES EM ÁREA DE TRANSIÇÃO FLORESTAL NO SUL DO BRASIL ${ }^{1}$
}

\author{
Elivane Salete Capellesso², Suéle Fatima Santolin² e Elisabete Maria Zanin ${ }^{2}$
}

\begin{abstract}
RESUMO - O banco e chuva de sementes podem ser indicadores utilizados para avaliar o potencial de recuperação de uma área que sofre influência antrópica. Assim, os objetivos deste trabalho foram quantificar o número de sementes viáveis e estimar a diversidade de espécies do banco e chuva de sementes, a fim de conhecer o potencial de recuperação de uma área inserida em uma matriz agrícola. Foram instalados 40 coletores para avaliar a chuva de sementes, bem como coletadas amostras de solo, para caracterizar o banco de sementes. Na chuva de sementes, foram encontradas 2.079 sementes, pertencentes a 43 espécies, sendo as famílias Lauraceae e Fabaceae as mais representativas. No banco de sementes, germinaram 69 indivíduos pertencentes a 23 espécies, sendo a família Asteraceae a mais representativa. Observou-se elevada diversidade de espécies na chuva de sementes $\left(H^{\prime}=2,34\right.$ nats ind $\left.^{-1}\right)$, em que as sementes das espécies secundárias tardias foram as mais abundantes, enquanto no banco de sementes, as espécies pioneiras. Em conclusão, o fragmento florestal encontrava-se em estágio sucessional avançado, o que pode garantir estabilidade ao sistema diante da influência antrópica.
\end{abstract}

Palavras-chave: Matriz agrícola; Estratégia de regeneração; Propágulos.

\section{SEED BANK AND SEED RAIN IN A TRANSITIONAL FOREST AREA IN THE SOUTH OF BRAZIL}

\begin{abstract}
The seed bank and seed rain can be used as indicators to evaluate the recovery potential of an anthropic area. Our aims were quantify the number of viable seeds and estimate species diversity of bank and seed rain to assess the recovery potential of an area surrounded by agriculture. We installed 40 collectors to evaluate seed rain and collected soil samples for seed bank quantifications. We found 2,079 seeds in seed rain, comprising 43 species, being Lauraceae and Fabaceae the most abundant families. Seed bank was represented by 69 seeds of 23 species, predominating Asteraceae family. We observed high species diversity in seed rain $\left(H^{\prime}=2.34\right.$ nats ind $\left.{ }^{-1}\right)$. Secondary species predominated in seed rain, while pioneer species in seed bank. In conclusion, forested area is in an advanced successional stage indicating a potential capacity of system under anthropogenic pressures.
\end{abstract}

Keywords: Agricultural matrix; Regeneration strategy; Propagules.

\footnotetext{
${ }^{1}$ Recebido em 04.03.2013 aceito para publicação em 16.06.2015.

${ }^{2}$ Universidade Regional Integrada do Alto Uruguai e das Missões, Programa de Pós-Graduação em Ecologia, Erechim, Rio Grande do Sul - Brasil.E-mail: <elivane_s_c@hotmail.com>, <emz@uri.com.br> e < suelesantolin@hotmail.com>.
} 


\section{INTRODUÇ̃̃O}

As florestas têm sido degradadas devido às atividades humanas (MIRANDA NETO et al., 2014), restando apenas pequenos remanescentes florestais nativos (FRANCO et al., 2012). A fragmentação e isolamento desses hábitats influenciam diretamente nas populações animais e vegetais, bem como nos processos ecossistêmicos como interações inseto-planta (AIZEN et al., 2002). Além disso, o isolamento dessas paisagens provocado pela urbanização, agricultura, entre outros fatores, pode ser considerado como agentes que dificultam a travessia de dispersores de um fragmento a outro devido, por exemplo, à existência de estradas, canais, falta de fontes de nutrição e condições microclimáticas desfavoráveis (TISCHENDORF; FAHRIG, 2000). Dessa maneira, a maior distância entre os fragmentos causaria aumento da mortalidade durante a dispersão (TISCHENDORF; FAHRIG, 2000), redução na taxa de migração entre as manchas, diminuindo a colonização de pequenas e isoladas manchas de hábitat (BERGMAN; LANDIN, 2001).

O banco de sementes que se forma no solo de uma floresta contribui para o processo de restauração desses ambientes, o que o torna de grande importância para avaliar o potencial de recuperação do equilíbrio dinâmico na área (SCHMITZ, 1992; CALEGARI et al., 2013). Através do banco de sementes, pode-se avaliar o estoque de sementes viáveis no solo (GARWOOD, 1989), podendo este ser transitório ou persistente, ou seja, pode haver germinação imediatamente ou ser adiada por período indefinido (SIMPSON, 1989; NÓBREGA et al., 2009). Esse sistema é dinâmico, com entrada de sementes por meio da dispersão e da chuva de sementes e saída, através da germinação, predação ou morte da semente (MESQUITA et al., 2014). Essas vias de entrada e saída são de grande importância, pois através da germinação podem substituir as plantas adultas que desaparecem dos fragmentos (CALEGARI et al., 2013), refletindo a composição florística do local (FENNER, 1985).

No solo pode ser encontrado grande número de sementes, mas somente pequena fração germina para dar origem às plântulas (HARPER, 1977), sendo o fluxo de entradas e saídas de sementes o principal determinante da potencialidade de estabelecimento da população naquele local (LOPES et al., 2010). Assim, a regeneração e crescimento de plantas estão relacionados com a chuva de sementes, sendo determinada pela produção e densidade da chuva de sementes e pela dinâmica do banco de sementes no solo (SIMPSON, 1989). A dispersão pela chuva de sementes é considerada a fase inicial na organização da dinâmica de florestas (HARDESTY; PARKER, 2002), permitindo que os indivíduos mortos sejam substituídos por espécies que se encontram no banco de sementes e de plântulas no solo(CAMPOS; SOUZA, 2003). Entretanto, o tamanho do fragmento, bem como a distância entre os fragmentos, pode influenciar a disponibilidade de sementes (TERBORGH, 1986), levando a mudanças na composição da chuva de sementes (FREITAS et al., 2013). A dispersão está ligada ao ciclo reprodutivo das plantas adultas, causando efeitos profundos na estrutura da vegetação (DU et al., 2007).

A grande maioria dos trabalhos de chuva e o banco de sementes foram desenvolvidos em áreas degradadas, de pastagem ou reflorestadas (MARTINS et al., 2008; NÓBREGA et al., 2009; RODIGUES et al., 2010; MIRANDA NETO et al., 2014). Assim, estudos envolvendo banco e chuva de sementes em fragmentos florestais que sofreram influência antrópica das áreas circundantes, como para a agricultura, carecem de trabalhos que identifiquem o potencial de recuperação da área diante de distúrbios ambientais que possam ocorrer ao longo do tempo. Diante disso, os objetivos foram quantificar o número de sementes viáveis e estimar a diversidade de espécies do banco e da chuva de sementes, a fim de conhecer o potencial de recuperação de uma área inserida em uma matriz agrícola.

\section{MATERIAL E MÉTODOS}

\section{1. Área de estudo}

A área de estudo está localizada em um fragmento florestal pertencente ao Horto Florestal Municipal de Erechim, que está situada a $768 \mathrm{~m}$ acima do nível do mar, na região Norte do Rio Grande do Sul, entre as coordenadas $27^{\circ} 42^{\prime} 41^{\prime \prime}$ e $27^{\circ} 43^{\prime} 15^{\prime \prime}$ de Latitude Sul e $52^{\circ} 18^{\prime} 48^{\prime \prime}$ e $52^{\circ} 17^{\prime} 53^{\prime \prime}$ de Longitude Oeste, com área aproximada de 60 ha. A área de estudo está inserida em uma matriz predominantemente agrícola, caracterizada por incluir um fragmento de vegetação natural numa transição entre floresta ombrófila mista e floresta estacional (BERNARDI; BUDKE, 2010). O clima da região é do tipo Cfa, que, de acordo com a classificação de Köppen, tem clima subtropical com temperatura média 
anual de $18,7^{\circ} \mathrm{C}$, com máximas e mínimas de $25,5^{\circ} \mathrm{C}$ (no mês de junho) e $12,7^{\circ} \mathrm{C}$ (no mês de janeiro). As chuvas são bem distribuídas ao longo do ano e atingem precipitação média de $1.912,3 \mathrm{~mm}^{-1} \mathrm{ano}^{-1}$ (BERNARDI; BUDKE, 2010).

\subsection{Coleta de dados}

Para caracterizar a densidade e composição do banco de sementes do solo, foram coletadas 40 amostras de solo, com distância mínima de $150 \mathrm{~m}$ entre os pontos de coleta. Cada amostra de solo foi retirada a cerca de $5 \mathrm{~cm}$ de profundidade (incluindo a serrapilheira). A delimitação das amostras foi realizada com o uso de gabarito de madeira $(0,3 \times 0,3 \mathrm{~m} \times 0,5 \mathrm{~cm})$, colocado sobre a superfície do solo, permitindo a padronização da área. A coleta das amostras foi realizada com o auxílio de uma pá de corte $(20 \times 20 \times 5 \mathrm{~cm})$. As 40 amostras de solo retiradas do banco de sementes do solo foram transferidas para casa de vegetação e alocadas em bandejas de $15 \mathrm{~cm}$ de profundidade. A temperatura média da casa de vegetação foi de $25^{\circ} \mathrm{C}$, cujas bandejas receberam irrigação diariamente durante o período de germinação, e o solo foi revolvido após o terceiro mês de coleta.

Para a coleta da chuva de sementes foram instalados 40 coletores nos mesmos locais utilizados para o banco de sementes no solo, os quais (molduras de madeira de $1 \mathrm{~m}^{2}$, revestidas com malha de náilon de $1 \mathrm{~mm}$ ) foram fixados a $50 \mathrm{~cm}$ do solo (ARAÚJO et al., 2004). Os propágulos foram coletados entre os meses de janeiro e junho, durante seis meses, assim como no estudo realizado por Caldato et al. (1996). Os meses de coleta são aqueles com maior dispersão no número de espécies, bem como no número de diásporos, segundo levantamento realizado por Araújo et al. (2004).

As plântulas do banco de sementes e as sementes oriundas da chuva de sementes foram classificadas até o nível taxonômico de espécie, de acordo com Lorenzi (1990; 2000ab) e Kissmann (1997) e com o auxílio de profissional especializado. As espécies vegetais foram classificadas quanto à forma de vida (arbórea, herbácea ou liana), estratégia de dispersão segundo definições de van der Pijl (1972) e estratégias de regeneração segundo Hartshorn (1980) e Clark e Clark (1987).

\subsection{Análise de dados}

A análise do banco de sementes foi realizada utilizando o método de germinação de Brown (1982).
A contagem das plantas foi realizada a cada 15 dias, por um período de seis meses.

A partir das plântulas germinadas e das sementes amostradas, foi avaliada a diversidade de espécies do banco e chuva de sementes, bem como calculado o índice de diversidade de Shannon-Wiener $\left(\mathrm{H}^{\prime}\right)$ e equabilidade de Pielou (J') no Programa Past ${ }^{\circledR}$.

\section{RESULTADOS}

Durante o período de seis meses em que o banco de sementes permaneceu na casa de vegetação, foram identificadas 23 espécies num total de 69 indivíduos regenerantes. As espécies foram distribuídas em 13 famílias botânicas (Tabela 1), sendo as mais representativas: Asteraceae (cinco espécies), Euphorbiaceae (quatro) e Rutaceae (três). Nas famílias Cannabaceae e Sapindaceae, observaram-se duas espécies; nas demais famílias, apenas uma espécie cada, sendo Acanthospermum hispidum DC. e Trema micrantha (L.) Blume aquelas com maior abundância de indivíduos.

No banco de sementes, pôde ser evidenciado o domínio das espécies pioneiras em relação às secundárias iniciais e tardias. Quanto à forma de vida, não houve predomínio de indivíduos do tipo arbóreo, em comparação com herbáceas e lianas.

Na avaliação da chuva de sementes, identificaramse 43 espécies, em um total de 2.079 propágulos, mas 17 sementes não foram classificadas no nível taxonômico de espécie (12 morfoespécies), pois não germinaram para a identificação da planta em razão, provavelmente, da dormência ou do tempo em que as sementes permaneceram nos coletores, inviabilizando-as. As espécies identificadas estão distribuídas em 18 famílias botânicas, sendo as mais representativas: Lauraceae e Fabaceae (cinco espécies cada), seguidas por Euphorbiaceae (três), Primulaceae, Sapindaceae e Myrtaceae (duas) e as demais famílias com uma espécie cada (Tabela 2).

As espécies com o maior número de propágulos coletados foram Cupania Vernalis Cambess., Gymnanthes concolor Spreng., Nectandra grandiflora Nees, Nectandra Lanceolata Nees, Nectandra megapotamica (Spreng.) Mez, Ocotea diospyrifolia (Meisn.) Mez, Pilocarpus penatifolius Lem., Sapium glandulosum (L.) Morong, e Thinouia mucronata Radlk., com mais de 100 propágulos cada uma, representando cerca de $90,47 \%$ do total de propágulos coletados. Assim,

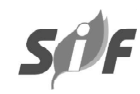

Revista Árvore, Viçosa-MG, v.39, n.5, p.821-829, 2015 
a diversidade de Shannon ( $\left.\mathrm{H}^{\prime}\right)$ foi de 2,34 nats ind $^{-1}$ e a equabilidade de Pilou (J'), 0,64.

A maioria das espécies localizadas foi classificada como arbóreas e, quanto à estratégia de regeneração, predominaram as espécies secundárias tardias. Na chuva de sementes, o maior número de propágulos coletados foi no mês de maio (443) e o menor, no mês de junho (222), enquanto no mês de janeiro se constatou maior número de espécies (19) e em junho, menor (10) (Figura 1).

\section{DISCUSSÃO}

A baixa densidade no banco de sementes, quando comparada com a chuva de sementes, pode ser explicada pela possível presença de um banco de sementes temporário, que funciona como estratégia de renovação da área (GRIME, 1979). Além disso, a densidade de sementes no solo de florestas tropicais pouco perturbadas tende a ser mais baixa, quando comparada com aquela de estádios de sucessão intermediária, sugerindo

Tabela 1 - Diversidade do banco de sementes do Horto Florestal Municipal de Erechim, RS. Estratégia de Regeneração (ER): pioneira (P), secundária tardia (St), secundária inicial (Si); e forma de vida (FV): arbórea (Arb), herbácea (Her) e liana (Lia). (*) Sem dados disponíveis na literatura.

Table 1 - Diversity of Seed Bank - Horto Florestal Municipal de Erechim/RS. Regeneration strategy (RS): Pioneer (P), late secondary (St), early secondary (Si), and life form (LF): arboreal (Arb), herbaceous (Her) and liana (Lia). (*) No data available in literature.

\begin{tabular}{|c|c|c|c|}
\hline FAMÍLIA/Espécie & Nome Popular & F.V. & E. R. \\
\hline \multicolumn{4}{|l|}{ AMARANTHACEAE } \\
\hline Amaranthus ssp. & & Caruru & HerP \\
\hline \multicolumn{4}{|l|}{ ARISTOLOCHIACEAE } \\
\hline \multicolumn{4}{|l|}{ ASTERACEAE } \\
\hline Acanthospermum hispidum DC. & Carrapicho-de-carneiro & Her & $\mathrm{P}$ \\
\hline Baccharis dracunculifolia DC. & Vassourinha & Her & $\mathrm{P}$ \\
\hline Conyza canadensis (L.) Cronq. & Voadeira & Her & $\mathrm{P}$ \\
\hline Eupatorium macrocephalum Less. & Eupatório & Her & $\mathrm{Si}$ \\
\hline Vernonia discolor (Spreng.) Less. & Vassourão-branco & Arb & $\mathrm{P}$ \\
\hline \multicolumn{4}{|l|}{ CANNABACEAE } \\
\hline Celtis iguanaea (Jacq.) Sarg. & Esporão-de-galo & Arb & $\mathrm{P}$ \\
\hline Trema micrantha (L.) Blume & Grandiúva & Arb & $\mathrm{P}$ \\
\hline \multicolumn{4}{|l|}{ COMMELINACEAE } \\
\hline Tradescantia fluminensis Vell. & Trapoeraba & Her & $\mathrm{P}$ \\
\hline \multicolumn{4}{|l|}{ CONVOLVULACEAE } \\
\hline Ipomoea grandifolia (Dammer) O’Donell & Corda-de-viola & Her & $\mathrm{P}$ \\
\hline \multicolumn{4}{|l|}{ EUPHORBIACEAE } \\
\hline Chamaesyce prostrata (Aiton) Small & Quebra-pedra-rasteira & Her & $\mathrm{P}$ \\
\hline Gymnanthes concolor Spreng. & Laranjeira-do-mato & Arb & $\mathrm{St}$ \\
\hline Sapium glandulosum (L) Morong & Pau-de-leite & Arb & $\mathrm{P} / \mathrm{Si}$ \\
\hline Sebastiania commersoniana (Baill.) L.B. Sm. \& Downs & Branquilho & Arb & $\mathrm{P}$ \\
\hline \multicolumn{4}{|l|}{ FABACEAE } \\
\hline Dalbergia frutescens (Vell.) Britton & Rabo-de-bugiu & Lia & $\mathrm{P}$ \\
\hline \multicolumn{4}{|l|}{ PTERIDACEAE } \\
\hline Doryopteris ssp & - & Her & $\mathrm{P}$ \\
\hline \multicolumn{4}{|l|}{ RUTACEAE } \\
\hline Pilocarpus pennatifolius Lem. & Jaborandi & Arb & $\mathrm{St}$ \\
\hline Zanthoxylum fagara (L.) Sarg. & Mamica-de-cadela & Arb & $\mathrm{Si}$ \\
\hline Zanthoxylum rhoifolium Lam. & Tambatá & Arb & $\mathrm{Si}$ \\
\hline \multicolumn{4}{|l|}{ SALICACEAE } \\
\hline Casearia sylvestris $\mathrm{Sw}$. & Chá-de-bugre & Arb & $\mathrm{P} / \mathrm{Si}$ \\
\hline \multicolumn{4}{|l|}{ SAPINDACEAE } \\
\hline Cardiospermum halicacabum $\mathrm{L}$. & Chumbinho & Her & $\mathrm{P}$ \\
\hline \multicolumn{4}{|l|}{ SOLANACEAE } \\
\hline Solanum mauritianum Scop. & Fumo-bravo & Arb & $\mathrm{St}$ \\
\hline
\end{tabular}

Revista Árvore, Viçosa-MG, v.39, n.5, p.821-829, 2015 
Tabela 2 - Diversidade de espécies da chuva de sementes - Horto Florestal Municipal de Erechim, RS. Estratégia de regeneração (ER): pioneira $(\mathrm{P})$, secundária inicial $(\mathrm{Si})$ e secundária tardia $(\mathrm{St}) .{ }^{*} \mathrm{Sem}$ dados disponíveis na literatura; forma de vida (FV): herbácea (Her), arbórea (Arb) e liana (Lia).

Table 2 -Diversity of rain seed species - Horto Florestal Municipal de Erechim/RS. Regeneration strategy (RS): Pioneer $(P)$, early secondary (Si), late secondary (St). * No data available in literature; life form (LF): herbaceous (Her), arboreal (Arb), and liana (Lia).

\begin{tabular}{|c|c|c|c|}
\hline Família/Espécie & Nome Popular & E. R. & F.V. \\
\hline \multicolumn{4}{|l|}{ APOCYNACEAE } \\
\hline Aspidosperma australe müll.arg. & \multirow[t]{2}{*}{ Peroba } & Arb & $\mathrm{P}$ \\
\hline ARECACEAE & & & \\
\hline $\begin{array}{l}\text { Syagrus romanzoffiana (Cham.) Glassman } \\
\text { BIGNONIACEAE }\end{array}$ & Coqueiro & Arb & $\mathrm{Si}$ \\
\hline $\begin{array}{l}\text { Jacaranda micrantha } \text { Cham. } \\
\text { BORAGINACEAE }\end{array}$ & Caroba & Arb & $\mathrm{Si}$ \\
\hline $\begin{array}{l}\text { Cordia americana (L.) Gottshling \& J.E.Mill. } \\
\text { CANNABACEAE }\end{array}$ & Guajuvira & Arb & $\mathrm{Si}$ \\
\hline $\begin{array}{l}\text { Celtis ehrenbergiana (Klotzsch) Liebm. } \\
\text { COMBRETACEAE }\end{array}$ & Teleira & Arb & $\mathrm{P}$ \\
\hline $\begin{array}{l}\text { Combretum fruticosum (Loefl.) Stuntz } \\
\text { EUPHORBIACEAE }\end{array}$ & Escova-de-macaco & Her & - \\
\hline Gymnanthes concolor Spreng. & \multirow{2}{*}{$\begin{array}{l}\text { Laranjeira-do-mato } \\
\text { Pau-de-leite }\end{array}$} & Arb & St \\
\hline Sapium glandulosum (L.) Morong & & Arb & $\mathrm{P}$ \\
\hline $\begin{array}{l}\text { Sebastiania brasiliensis Spreng. } \\
\text { FABACEAE }\end{array}$ & Leiteiro & \multicolumn{2}{|l|}{ Arb } \\
\hline Apuleia leiocarpa (Vogel) J.F.Macbr. & Grápia & Arb & St \\
\hline Dalbergia frutescens (Vell.) Britton. & Rabo-de-búgiu & Lia & $\mathrm{Si}$ \\
\hline Inga vera Willd. & Ingá-banana & Arb & $\mathrm{Si}$ \\
\hline Machaerium paraguariense Hassl. & Farinha-seca & Arb & $\mathrm{Si}$ \\
\hline $\begin{array}{l}\text { Parapiptadenia rigida (Benth.) Brenan } \\
\text { LAURACEAE }\end{array}$ & Angico-vermelho & Arb & St \\
\hline Nectandra grandiflora $\mathrm{Nees}$ & Canela-fedida & Arb & St \\
\hline Nectandra lanceolata $\mathrm{Nees}$ & Canela-fedida & Arb & St \\
\hline Nectandra megapotamica (Spreng.) Mez & Canela-amarela & Arb & St \\
\hline Ocotea diospyrifolia (Meisn.) Mez & Canela & Arb & St \\
\hline $\begin{array}{l}\text { Ocotea puberula (Rich.) Nees } \\
\text { LOGANIACEAE }\end{array}$ & Canela-guaiacá & Arb & St \\
\hline $\begin{array}{l}\text { Strychnos brasiliensis (Spreng.) Mart. } \\
\text { MYRTACEAE }\end{array}$ & Esporão-de-galo & Arb & $\mathrm{Si}$ \\
\hline $\begin{array}{l}\text { Campomanesia xanthocarpa O.Berg } \\
\text { PHYTOLACCACEAE }\end{array}$ & Guabiroba & Arb & St \\
\hline $\begin{array}{l}\text { Seguieria aculeata Jacq. } \\
\text { PICRAMNIACEAE }\end{array}$ & - & Arb & $\mathrm{Si}$ \\
\hline Picramnia parvifolia Engl. & Pau-amargo & Arb & St \\
\hline $\begin{array}{l}\text { PINACEAEPinus elliottii Engel. } \\
\text { PRIMULACEAE }\end{array}$ & Pinheiro-americano & Arb & $\mathrm{P}$ \\
\hline Myrsine parvifolia DC. & Capororoquinha & Arb & $\mathrm{P}$ \\
\hline $\begin{array}{l}\text { Myrsine umbellata } \text { Mart. } \\
\text { ROSACEAE }\end{array}$ & Capororoca & Arb & $\mathrm{P}$ \\
\hline $\begin{array}{l}\text { Prunus myrtifolia (L.) Urb. } \\
\text { RUTACEAE }\end{array}$ & Pessegueiro-do-mato & Arb & $\mathrm{P}$ \\
\hline Balfourodendron riedelianum (Engl.) Engl. & & & \\
\hline $\begin{array}{l}\text { Pilocarpus pennatifolius Lem. } \\
\text { SAPINDACEAE }\end{array}$ & GuatambuJaborandi & ArbArb & StSt \\
\hline Cupania vernalis Cambess. & Camboatá-vermelho- & Arb & $\mathrm{Si}$ \\
\hline Thinouia mucronata Radlk. & & Lia & - \\
\hline Morfoespécie 1 & - & - & - \\
\hline Morfoespécie 2 & - & - & - \\
\hline Morfoespécie 3 & - & - & - \\
\hline Morfoespécie 4 & - & - & - \\
\hline Morfoespécie 5 & - & - & - \\
\hline Morfoespécie 6 & - & - & - \\
\hline Morfoespécie 7 & - & - & - \\
\hline Morfoespécie 8 & - & - & - \\
\hline Morfoespécie 9 & - & - & - \\
\hline Morfoespécie 10 & - & - & - \\
\hline Morfoespécie 11 & - & - & - \\
\hline Morfoespécie 12 & - & - & - \\
\hline
\end{tabular}



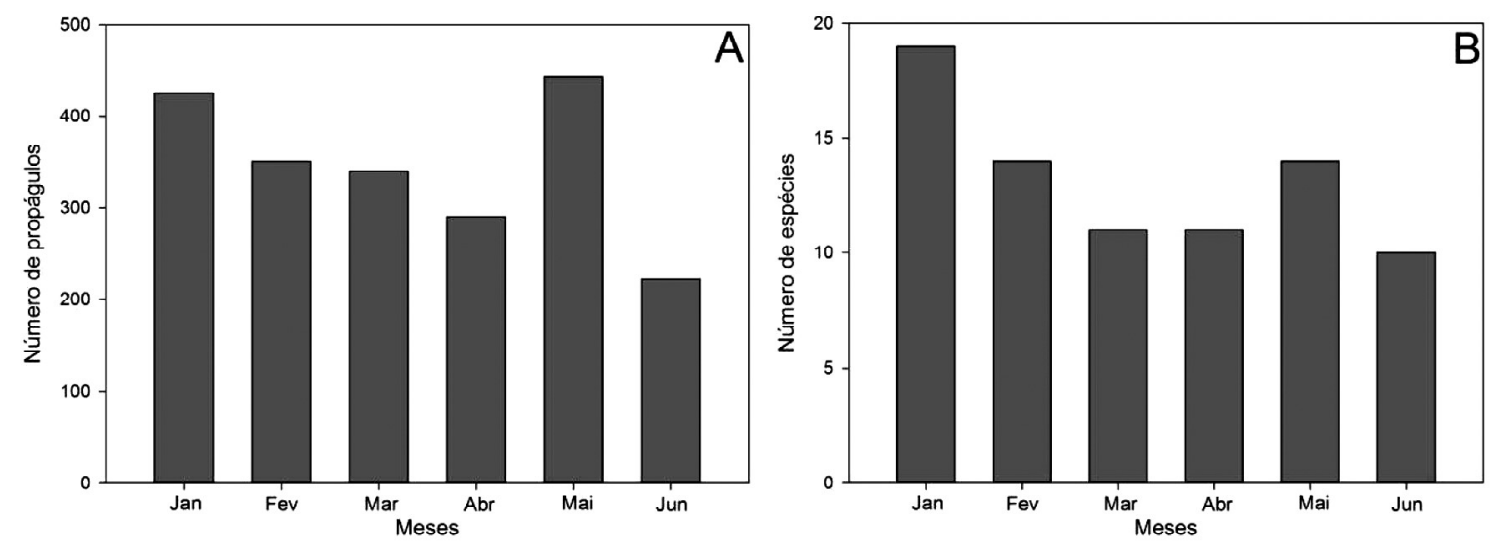

Figura 1 - Distribuição do número de propágulos (A) e espécies (B) amostrados ao longo dos meses na chuva de sementes no Horto Florestal Municipal de Erechim, RS

Figure 1 - Distribution of the number of propagules (A) and species (B) sampled over the months of seed rain in Horto Florestal Municipal de Erechim/RS.

decréscimo no estoque de sementes do solo com o decorrer do processo sucessional (BAIDER et al., 2001).

No fragmento estudado, verificou-se diversidade de espécies intermediárias no banco de sementes no solo, em comparação com outros levantamentos realizados (LONGHI et al., 2005; BATISTA NETO et al., 2007; BRAGA et al., 2008). As famílias mais abundantes neste estudo foram Asteraceae e Euphorbiaceae, assim como em levantamentos realizados por Sccoti et al. (2011), Braga et al. (2008) e Franco et al. (2012) em Viçosa, MG, quando observaram que a família Asteraceae foi a mais representativa. As espécies arbóreas foram as mais representativas neste artigo, diferindo dos outros estudos de Sccoti et al. (2011) e Franco et al. (2012), que relataram abundância maior de espécies herbáceas, mostrando a importância das espécies arbóreas na chuva de sementes do fragmento.

O banco de sementes no solo não reflete a riqueza arbórea do fragmento estudado. Grime (1979) e Garwood (1989) argumentaram que as espécies do banco de solo são, na maioria das vezes, raras ou até mesmo ausentes na floresta estabelecida, sendo provenientes de diferentes áreas e épocas. Com base nas estratégias de regeneração, a presença em grande número de espécies e indivíduos caracterizados como pioneiros (considerando herbáceas e arbóreas) no banco de sementes é semelhante ao observado por Mikich e Silva (2001), em estudo realizado em uma Floresta Estacional. Essas espécies iniciais de regeneração em uma sucessão ecológica desenvolvem-se em clareiras e nas bordas dos fragmentos, sendo dependentes de condições de maior luminosidade (GANDOLFI et al., 1995). Com isso, os indivíduos pioneiros facilitam o estabelecimento de outras espécies tolerantes à sombra, agindo como abrigo para os vetores de dispersão e fornecendo hábitats adequados ao recrutamento (BAIDER et al., 1999). Esse processo de regeneração é importante quando ocorrem distúrbios sobre o ecossistema, onde a presença de espécies herbáceas é fundamental para o restabelecimento das comunidades vegetais, consistindo em bom indicador de resiliência da área (MARTINS et al., 2008). Assim, caso venha ocorrer algum distúrbio no fragmento, as espécies pioneiras serão as primeiras a se estabelecerem, devido à sua exigência de luminosidade, seguida pelas espécies secundárias iniciais, as quais Souza et al. (2006) afirmaram ser as mais abundantes em levantamentos de banco de sementes. Mesmo em estágios sucessionais mais avançados, como é o caso do fragmento em estudo, as espécies pioneiras estão presentes e têm a função de auxiliar na recuperação de clareiras ocasionais no local (LONGHI et al., 2005).

As famílias mais abundantes na chuva de sementes foram Fabaceae e Lauraceae, sendo a Fabaceae também a mais abundante no trabalho de Campos et al. (2009), que avaliaram um fragmento em Viçosa, MG. Neste estudo, destaca-se a importância dessas famílias na dinâmica dos fragmentos, sendo as mais encontradas em estudos desenvolvidos na Região Sul do Brasil. 
A diversidade de espécies de sementes $\left(\mathrm{H}^{\prime}=2,34\right.$ nats ind ${ }^{-1}$ ) foi maior quando comparada com o trabalho de Silva et al. (2009), cuja diversidade foi de 1,69 nats ind $^{-1}$, em uma floresta de restinga em São Paulo. Além disso, Pivello e colaboradores (2006), estudando a diferença entre borda e interior de um fragmento em Cotia, SP, não observaram diversidade semelhante à do fragmento estudado.

O maior número de espécies ocorreu no mês de janeiro, pois foram encontradas 19 espécies, assim como em outros trabalhos que destacaram os meses de dezembro e janeiro, com dispersão de maior número de espécies (CALDATO et al., 1996; ARAÚJO et al., 2004). No entanto, o mês com maior número de propágulos dispersos foi maio (com 455), e a dominância referente ao mês pode variar de acordo com a predominância de alguma espécie nas coletas da chuva de sementes. Por exemplo, neste estudo, o pico de dispersão da espécie Thinouia mucronata foi no mês de maio, com 253 propágulos dispersos, influenciando as comparações mensais.

\section{CONCLUSÃO}

O banco e chuva de sementes apresentam elevada diversidade de espécies, em comparação com outros trabalhos. Além disso, o maior número de espécies pioneiras encontradas no banco de sementes indica que esse fragmento apresenta capacidade de autorrecuperação diante de distúrbio. Essas sementes podem estar sendo armazenadas no solo para que em momentos favoráveis, como na abertura de clareira, o banco consiga restabelecer a estrutura local. Entretanto, o maior número de espécies classificadas como secundárias tardias na chuva de sementes demonstra que o fragmento florestal se encontra em estágio sucessional avançado, o que pode garantir estabilidade ao sistema diante da influência antrópica.

\section{AGRADECIMENTOS}

Ao Laboratório de Geoprocessamento e Planejamento Ambiental da Universidade Regional Integrada do Alto Uruguai e das Missões (URI), Campus de Erechim, pelo apoio logístico; e à URI, pelo apoio financeiro; e à Prefeitura Municipal de Erechim, por meio da Secretaria Municipal de Meio Ambiente, pela permissão da coleta e da execução do trabalho no Horto Florestal Municipal.

\section{REFERÊNCIAS}

AIZEN, M. A.; VÁZQUEZ, D. P.; SMITH-

RAMÍREZ, C. Natural history and conservation of plant-animal mutualisms in the temperate forest of southern South America. Revista Chilena de Historia Natural, v. 75, n.1, p.79-97, 2002.

ARAÚJO M.M.; LONGHI, J.S.; BARROS, P.L.C.; BRENA, D.A. Caracterização da chuva de sementes, banco de sementes do solo e de plântulas em Floresta Estacional Decidual ripária Cachoeira do Sul, RS, Brasil. Scientia florestalis, v.66, p.128-141, 2004.

BAIDER, C.; TABARELLI, M.; MANTOVANI, W. O banco de sementes de um trecho de Floresta Atlântica Montana (São Paulo, Brasil). Revista Brasileira de Biologia, v.59, n.2, p.319-328, 1999.

BAIDER, C.; TABARELLI, M.; MANTOVANI, W. The soil seed bank during Atlantic Forest regeneration in southeast Brasil. Revista Brasileira de Biologia, v.61, n.1, p.35-44, 2001.

BATISTA NETO, J.P.; REIS M.G.F.; REIS, G.G.; SILVA, A.F.; CACAU, F.V. Banco de sementes do solo de uma Floresta Estacional Semidecidual, em Viçosa, Minas Gerais. Ciência Florestal, v.17, n.4, p.311-320, 2007.

BERGMAN, K.O.; LANDIN, J. Distribution of occupied and vacant sites and migration of Lopinga achine (Nymphalidae: Satyrinae) in a fragmented landscape. Biological

Conservation, v.102, n.1, p.183-190, 2001.

BERNARDI, S.; BUDKE, J.C. Estrutura da sinúsia epifítica e efeito de borda em uma área de transição entre Floresta Estacional Semidecídua e Floresta Ombrófila Mista. Floresta, n.40, p.8192, 2010.

BRAGA, A.J.T.; GRIFFITH, J.J.; PAIVA, H.N.; MEIRA NETO, J.A.A. Composição do banco de sementes de uma Floresta Secundária considerando o seu potencial de uso para recuperação Ambiental. Revista Árvore, v.32, n.6, p.1089-1098, 2008.

BROWN, D. Estimating the composition of a forest seed bank: a comparison of the seed

Revista Árvore, Viçosa-MG, v.39, n.5, p.821-829, 2015 
extraction and seedling emergence methods. Canadian Journal of Botany, v. 70 , n. 8 , p.1603-1612, 1982.

CALDATO, S.L.; FLOSS, P.A.; CROCE, D.M.; LONGHI, J.S. Estudo da regeneração natural, banco de sementes e chuva de sementes na reserva genética florestal de Caçador, SC. Ciência Florestal, v.6, n.1, p.27-38, 1996.

CALEGARI, L.; MARTINS, S.V.; CAMPOS, L.C.; SILVA, E.; GLERIANI, J.M. Avaliação do banco de sementes do solo para fins de restauração florestal em Carandaí, MG. Revista Árvore, v.37, n.5, p.871-880, 2013.

CAMPOS, E. P.; VIEIRA, M.F.; SILVA, A.F.; MARTINS, S.V.; CARMO, F.M.S.; MOURA, V.M.; RIBEIRO, A.S.S. Chuva de sementes em Floresta Estacional Semidecidual em Viçosa, MG, Brasil. Acta Botanica Brasílica, v.23, p.451-458, 2009.

CAMPOS, J.B.; SOUZA, M.C. Potencial for natural Forest regeneration from seed bank in an upper Paraná river floodplain, Brazil. Brazilian Archives of Biology and Technology, v.46, n.4, p.625-639, 2003.

CLARK, D.A.; CLARK, D.B. Análisis de La regeneración de árboles Del dosel en bosque muy húmedo tropical: aspectos teóricos y praticos. Revista de Biologia Tropical, v.35, n.1, p.41-54, 1987.

DU, X.; GUO, Q.; GAO, X.; MA,K. Seed rain, soil seed bank, seed loss and regeneration of Castanopsis fargesii (Fagaceae) in a subtropical evergreen broadleaved forest. Forest Ecology and Management, v.238, n.1-3, p.212-219, 2007.

FENNER, M. Seed ecology. London: Chapman \& Hall, 1985. 147 p.

FRANCO, B.K.S.; MARTINS, S.V.; FARIA, P.C.L.; RIBEIRO, G.A. Densidade e composição florística do banco de sementes de um trecho de Floresta Estacional Semidecidual no campus da Universidade Federal de Viçosa, Viçosa, MG. Revista Árvore, v.36, n.3, p.423-432, 2012.

FREITAS, C.G.; DAMBROS, C.; CAMARGO, J.L.C. Changes in see d rain across Atlantic Forest

Revista Árvore, Viçosa-MG, v.39, n.5, p.821-829, 2015 fragments in Nort heast Brazil. Acta

Oecologica, v.53, n.1, p.49-55, 2013.

GANDOLFI, S.; LEITÃO-FILHO, H.F.; BEZERRA, C.L. Levantamento florístico e caráter sucessional das espécies arbustivo-arbóreas de uma floresta mesófila semidecídua no município de Guarulhos, SP. Revista Brasileira de Biologia, v.55, n.4, p.753-767, 1995.

GARWOOD, N.C. Tropical soil seed banks: a rewiew. In: LECK, M.A.; PARKER, T.V.; SIMPSON. R.L. (Ed.) Ecology of soil seed banks. New York: Academic Press, 1989. p.149-209.

GRIME, J.P. Plant strategies and vegetation processes. New York: John Wiley \& Sons, 1979. $222 \mathrm{p}$.

HARDESTY, B.D.; PARKER, V.T. Community seed rain patterns and a comparison to adult community structure in a West African tropical forest. Plant Ecology, v. 164, n.1, p.49-64, 2002.

HARPER, J.L. Population biology of plants. London: Academic Press, 1977.

HARTSHORN, G.S. Neotropical forest dynamics. Tropical Succession, v.12, n.1, p.23-30, 1980.

KISSMANN, K.G.; GROTH, D. Plantas infestantes e nocivas. 2.ed. São Paulo: BASF, 1997. 3v.

LONGHI, S.J.; BRUN, E.J.; OLIVEIRA, D.M.; FIALHO, L.E.B.; WOJCIECHOWSKI, J.C.; VACCARO, S. Banco de sementes do solo em três fases sucessionais de uma Floresta Estacional Decidual em Santa Tereza, RS. Ciência Florestal, v. 15, n.4, p.359-370, 2005.

LOPES, S.F.; OLIVEIRA, A.P.; NEVES, S.B.; SCHIAVINI, I. Dispersão de sementes de uruvalheira (Platypodium elegans VOG.) (Fabaceae) em um cerradão, Uberlândia-MG. Revista Árvore, v.34, n.5, p.807-813, 2010.

LORENZI, H. Manual de identificação e controle de plantas daninhas: plantio direto e convencional . 3.ed. Nova Odessa: Instituto Plantarum, 1990. 383p.

LORENZI, H. Plantas daninhas do

Brasil: terrestres, aquáticas, parasitas e tóxicas. 
3.ed. Nova Odessa: Instituto Plantarum, 2000a. $608 \mathrm{p}$.

LORENZI, H. Árvores brasileiras: manual de identificação e cultivo de plantas arbóreas do Brasil. 3.ed. Nova Odessa: Instituto Plantarum, 2000b. 352p.

MARTINS, S.V.; ALMEIDA, D.P.; FERNANDES, L.V.; RIBEIRO, T.M. Banco de sementes como indicador de restauração de uma área degradada por mineração de caulim em Brás Pires, MG. Revista Árvore, v.32, n.6, p.1081-1088, 2008.

MESQUITA, M.L.R.; ANDRADE, L.A.; PEREIRA, W.E. Banco de sementes do solo em áreas de cultivo de subsistência na floresta ombrófila aberta com babaçu (orbygnia phalerata mart.) no Maranhão. Revista Árvore, v.38, n.4, p.677-688, 2014.

MIKICH, S.B.; SILVA, S.M. Composição florística e fenologia das espécies zoocóricas de remanescentes de Floresta Estacional Semidecidual no Centro-oeste do Paraná, Brasil. Acta Botanica Brasílica, v.15, n.1, p.89113, 2001.

MIRANDA NETO, A.M.; MARTINS, S.V.; SILVA, K.A.; GLERIANI, J.M. Banco de sementes do solo e serapilheira acumulada em floresta restaurada. Banco de sementes do solo e serapilheira acumulada em floresta restaurada. Revista Árvore, v.38, n.4, p.609-620, 2014.

NÓBREGA, A.M.F.; VALERI, S.V.; PAULA, R.C.; PAVANI, M.C.M.D.; SILVA, S.A. Banco de sementes de remanescentes naturais e de áreas reflorestadas em uma várzea do rio Mogi-GuaçuSP. Revista Árvore, v.33, n.3, p.403-411, 2009.

PIVELLO, V.R.; PETENON, D.; JESUS, F.M.; MEIRELLES, S.T.; VIDAL, M.M.; ALONSO, R.A.S.; FRANCO, G.A.D.C.; METZGER, J.P. Chuva de sementes em fragmentos de Floresta Atlântica (São Paulo, SP, Brasil), sob diferentes situações de conectividade, estrutura florestal e proximidade da borda. Acta Botanica

Brasílica, v.20, n.4, p.845-859, 2006.

RODRIGUES, B.D.; MARTINS, S.V.; LEITE, H.G. Avaliação do potencial da transposição da serapilheira e do banco de sementes do solo para restauração florestal em áreas degradadas.

Revista Árvore, v.34, n.1, p.65-73, 2010.

SCCOTI, M.S.V.; ARAUJO, M.M.; WENDLER, C.F.; LONGHI, J.S. Mecanismos de regeneração natural em remanescente de Floresta Estacional Decidual. Ciência Florestal, v.21, n.3, p.459472,2011 .

SILVA, C.R.; PAOLI, A.A.S.; BARBOSA, J.M.; SANTOS JUNIOR, N.A. Chuva de sementes em uma Floresta Alta de Restinga em Ilha Comprida (SP). Cerne, v. 15, n.3, p.355-365, 2009.

SIMPSON, R.L. Ecology of soil seed bank, San Diego: Academic Press, 1989.

SCHIMTZ, M.C. Banco de sementes no solo em áreas do reservatório da UHE Paraibuna. In: KAGEYAMA, P.Y. Recomposição da vegetação com espécies arbóreas nativas em reservatórios de usinas hidrelétricas da CESP. Série IPEF, v.8, n.25, p.7-8, 1992.

SOUZA, P.A.; VENTURIN, N.; GRIFFITH, J.J.; MARTINS, S.V. Avaliação do banco de sementes contido na serapilheira de um fragmento florestal visando recuperação de áreas degradadas.

Cerne, v. 12, n.1, p.56-67, 2006.

TERBORGH, J. Keystone plant resources in the tropical forest. In: SOULÉ, M.E. (Ed.)

Conservation biology: the science of scarcity and diversity. Sinauer, Sunderland: 1986. p.330-344.

TISCHENDORF, L.; FAHRIG, L. On the usage and measurement of landscape connectivity. Oikos, v.90, n.1, p.7-19, 2000.

van der PIJL, L. Principles of dispersal in higher plants. 2.ed. New York: SpringerVerlag, 1972. $162 \mathrm{p}$. 